\title{
Sleep in adolescents and young adults
}

\author{
Authors: Ellen S Bruce, ${ }^{A}$ Laura Lunt ${ }^{B}$ and Janet E McDonagh ${ }^{C}$
}

Sleep has an important role in maintaining health and wellbeing; this relationship is becoming increasingly recognised for adolescents and young adults. Many physicians will encounter young people who present with complaints or conditions that have some relation to poor sleep. This review article looks at why sleep matters within this population group, how it can impact on longer term health consequences and discusses some tools to help enable the clinician to evaluate and address sleep within clinical practice.

KEYWORDS: Adolescents, chronic disease, fatigue, sleep, young adults

\section{Introduction}

The important role of sleep in maintaining health and wellbeing has become increasingly recognised. Sleep has many functions (including promoting growth, learning and cognitive development), ${ }^{1}$ has a role in immunity ${ }^{2}$ and studies have reported an association between poor sleep and heart disease in adults. ${ }^{3,4}$ Its relevance is now becoming increasingly recognised in adolescents and young adults, particularly with regards to our 24-hour culture of connectivity and media consumption. ${ }^{5,6}$

The 2014 Health behaviour in school-aged children report demonstrated that for 15-year-olds in England, 30\% of boys and $49 \%$ of girls reported experiencing sleeping difficulties and $36 \%$ of 15 -year-olds reported not having enough sleep to be able to concentrate on school work. ${ }^{7}$ Poor sleep in

\footnotetext{
Authors: ${ }^{\text {A }}$ consultant rheumatologist, Central Manchester University Hospitals NHS Foundation Trust, Manchester, UK; ${ }^{B}$ adolescent and young adult rheumatology research assistant, Arthritis Research UK Centre for Epidemiology, Centre for Musculoskeletal Research, Manchester Academic Health Science Centre, University of Manchester and NIHR Manchester Biomedical Research Centre, Central Manchester NHS Foundation Trust, Manchester Academic Health Science Centre, Manchester, UK; ${ }^{C}$ consultant in paediatric and adolescent rheumatology, Central Manchester University Hospitals NHS Foundation Trust, Arthritis Research UK Centre for Epidemiology, Centre for Musculoskeletal Research, Manchester Academic Health Science Centre, University of Manchester and NIHR Manchester Biomedical Research Centre, Central Manchester NHS Foundation Trust, Manchester Academic Health Science Centre, Manchester, UK
}

adolescents and young adults can result in longer-term sleep problems, which may impact on them into adulthood. In one study, adolescent sleep disturbances predicted adult sleep disturbances. ${ }^{8}$ If problems were present at age 16 years, a third still had problems at 23 years and $10 \%$ at 42 years.

Many adult physicians will encounter young people who might present with complaints or conditions that have some relation to poor sleep and/or fatigue. If sleep is not first considered by the physician, this is likely to remain unrecognised and consequently result in ineffective treatment strategies if the underlying problem is not addressed. Additionally, it may be that if further questioned, a complaint about sleep disturbance may reveal another concern, or hidden agenda. In this article we will look at sleep in the adolescent and young adult (AYA) population, why it matters and what to do about it.

\section{The biology of adolescent sleep}

Adolescents and young adults have been defined by the World Health Organization as those aged between 10 and 24 years. During this period of development and maturation, there are a number of changes taking place, with brain maturation continuing throughout this period. ${ }^{9}$ It is therefore not surprising that the biology and pattern of sleep differs within this age range.

Adolescents are recognised to have a biological delay in the timing of sleep onset, which can result in them staying awake later. This is more often associated with older adolescents as demonstrated in a number of studies undertaken in this population. ${ }^{10}$ The reason for this is a change in the two processes that are involved in sleep regulation; the intrinsic circadian timing system and the homeostatic sleep-wake system. ${ }^{11}$

A reduced urge to fall asleep results in adolescents staying awake later; hence, they have an overall shortened sleep duration. This factor is particularly noticeable during the school years, as the time required to rise for the school day remains constant. However, the amount of sleep required by this population group has not reduced, resulting in a period of relative sleep deprivation compared with the adult population. $^{10}$

\section{Adolescent sleep disorders}

There are of course specific sleep disorders that affect AYAs, the most common being delayed sleep phase syndrome. This develops in adolescence and can continue into young 
adulthood; it is reported to affect 7\% of adolescents. Delayed sleep phase syndrome is a pathological shift of the normal delay in the timing of sleep onset that occurs at this age, those affected will typically go to bed between 1am and $4 \mathrm{am}$ and wake much later in the morning. ${ }^{12}$ This results in significant sleep deprivation if the individual's daily routines do not allow for a late awakening. Treatment requires gradual adjustment of the sleep cycle and use of bright light therapy in the morning with avoidance of bright light in the evening. ${ }^{12,13}$ Other less common sleep disorders include night terrors, sleep walking, sleep-onset anxiety, restless legs and narcolepsy, but these are beyond the scope of this article. ${ }^{13}$

\section{The relationship between sleep and health}

It is important to note that there is often a bidirectional relationship between sleep and health and having an awareness of the complexity of this relationship is helpful when assessing sleep in clinical practice. An early indication of a patient developing problems with their sleep can be sleep fragmentation, where individuals have difficulty remaining asleep, resulting in them feeling unrefreshed. This sleep fragmentation may then lead onto further health consequences of poor sleep, including daytime somnolence, impaired cognition and poor mood.

Sleep fragmentation can itself occur because of an underlying health condition, such as obstructive sleep apnoea and it is this bidirectional relationship that requires consideration. Additionally, this interplay between sleep and health in AYAs can have a continuing impact into adulthood and it is therefore of importance to all physicians who encounter this patient cohort. This is particularly true of young people with longterm health conditions. Some of the important health-related associations are discussed here.

\section{Sleep and obesity}

Within the last decade it has been demonstrated that shorter sleep duration, or inadequate sleep, is associated with the development of obesity. Of note, this association is stronger for both children and young adults compared with older adults. ${ }^{14,15}$ Adolescents who have shorter sleep durations have also been shown more likely to be overweight. ${ }^{16,17}$ However, causation is difficult to determine because of the fact that most of the epidemiological studies are observational. There are also confounding factors to consider. For example, spending a lot of time inactive, accessing media or entertainment can result in both a shorter sleep duration and also be associated with later obesity through other associations of a sedentary lifestyle.

Possible physiological reasons for this association could be explained by the fact that glucose metabolism and the hunger hormones (leptin and ghrelin) follow certain nocturnal patterns and disruption to this, by shortening the duration of sleep, has an impact on calorie intake and utilisation. ${ }^{18,19}$

Obesity in AYAs and in later life is known to be associated with chronic health problems, such as type 2 diabetes and hypertension. An additional consideration is the relationship between obesity and obstructive sleep apnoea and the resultant impairment on sleep. Therefore, the association between sleep and obesity has bidirectional importance.

\section{Sleep and mental health}

Adolescents do not differ from adults in terms of the effect of depression on sleep. Similarly, anxiety disorders also impact on sleep although, again, it is important to note that causality is not clear in this relationship. ${ }^{20,21}$ For example, a complaint of poor sleep may be a symptom of an underlying mood disorder and those with depression may have an altered self-recall of duration or quality of sleep. In turn, a shorter sleep duration might be a risk factor for developing depression. ${ }^{22}$

The last decade has seen increasing interest in the relationship between sleep problems in adolescence and the development of risk-taking behaviour. Studies undertaken in American 14-18-year-olds have demonstrated an association between insufficient sleep and an increased likelihood of engaging in some health-risk behaviours, such as smoking, alcohol use, marijuana use and violence. ${ }^{23,24}$ In a Finnish study of 13-18-year-olds, $27 \%$ reported weekly sleep problems, which in turn were associated with older individuals as well as peer and alcohol problems. ${ }^{25}$ One theory as to why this association might exist is that sleep deprivation affects cognition, and therefore poor choices may result from peer pressure or an inability to adequately assess the consequence of these behaviours. In this instance, it is the risk-taking behaviour that may lead to both short- and long-term health problems that can continue into adulthood.

\section{Sleep and pain}

There is an established link between poor sleep and pain in healthy populations. However, it has also been demonstrated in conditions such as juvenile idiopathic arthritis, headaches or as part of a pain syndrome (such as chronic widespread pain and fibromyalgia). ${ }^{26}$ Studies focusing on pain and these clinical conditions have more often been separated into an adult or paediatric population, neglecting the fact that AYAs are a discrete population with specific developmental processes that are often overlooked.

The relationship between headaches and sleep is well known. Disturbances in normal sleep, in terms of either quality or duration, can exacerbate or even trigger headaches. There is also thought to be an association with sleep disorders in earlier childhood and later presentation of migraines or headaches. ${ }^{27}$ Although most studies have not focused on a specific AYA population, those that have also demonstrated an interaction or association between headaches and sleep. ${ }^{28-30}$

The relationship between sleep and pain for young people with juvenile idiopathic arthritis and other rheumatological conditions has more recently been explored. Most of the reported studies use either parent-proxy questionnaires or a combination of patient and parent-proxy questionnaires and the majority of studies have a mean age of less than 13 years. ${ }^{31,32}$ As such, they are not representative of the age range included in an AYA cohort. Although an association is demonstrated between sleep and pain in these studies, there are a number of other variables to consider that may not be as easily measured, such as psychological or social factors.

For patients of all ages with chronic pain, the importance of sleep is known to be an integral part of assessment and treatment strategies although, again, there is a paucity of studies assessing the impact on the AYA cohort. One study focusing 
on 12-18-year-olds demonstrated that the presence of chronic pain, rather than the intensity of pain, was predictive for insomnia and that those with chronic pain had a sixfold higher risk for insomnia symptoms. ${ }^{33}$

\section{Sleep and illness}

Adolescents and young adults are well represented within the acute hospital setting, with 16-19-year-olds accounting for $20 \%$ of inpatients. ${ }^{34}$ Sleep within a hospital environment is challenging for all patients, but AYAs may encounter additional difficulties with their sleep cycle not matching the traditional ward bedtime routine. Early wakening for observations, medicines and ward rounds can contribute to sleep deprivation. Being on an adult ward may result in exposure to older patients with significant illness and even death, the impact of which may affect the AYA's own cognitive concerns about their illness and can have a detrimental effect on their sleep.

Those AYAs who have a chronic illness will have more frequent encounters with their GPs and hospital physicians and it is therefore important to recognise that these patients may be encountering problems with sleep or fatigue. Most chronic illnesses have been recognised to impact on sleep - for example, asthma, cancer, epilepsy, kidney disease and sickle cell disease. ${ }^{35}$ The impact on an individual's sleep may be related to a multitude of causes, including disease-specific factors, medication side effects, hospital admissions and psychological factors. As already highlighted, these interactions can be bidirectional and are therefore important to recognise.

\section{Sleep and fatigue}

It is worth taking some time to consider whether there is any difference between feeling sleepy or fatigued, as the complaint of tiredness may be used by someone to describe either of these symptoms. Sleepiness relates to being more likely to fall asleep, whereas fatigue is a perception of low energy after otherwise normal activities. ${ }^{36}$ Understanding which symptom is being experienced can help with managing the underlying problem although it may at times be difficult to distinguish between the two.

Feeling fatigued during adolescence and young adulthood can be a short-lived complaint, particularly during this period of development where there are physical demands associated with growth and maturation along with social, educational and occupational demands. A smaller proportion will have chronic fatigue syndrome, defined as a persistent debilitating, severe fatigue of more than 3 months duration with associated symptoms such as joint pain, tenderness, headaches and unrefreshing sleep, which are not explained by an alternative diagnosis. A study undertaken with British 11-15-year-olds identified an incidence of $30.3 \%$ for fatigue and $0.5 \%$ for chronic fatigue syndrome. ${ }^{37} \mathrm{An}$ in-depth discussion about chronic fatigue syndrome in adolescents is beyond the scope of this article, but management requires a multidisciplinary and biopsychosocial approach to control symptoms. ${ }^{38}$

\section{Sleep and accidents}

UK data for 2010 reported over half of all deaths in 15-19-yearolds were attributable to external causes, including injuries and poisoning, risks and behaviours. Transport accidents were responsible for $77 \%$ of the deaths for those aged between 10 and 18 years. ${ }^{39}$ Sleep deprivation is likely to have a role here as transport data for the UK demonstrate that for sleep-related crashes, $38 \%$ of drivers are aged between 16 and 30 years. ${ }^{40}$ International data have shown that traffic-related mortality is higher in males and rose with increasing age within the AYA age group. ${ }^{41}$

\section{Assessing sleep in clinical practice}

The assessment of sleep requires an understanding of the many factors that can impact upon it, some of which have been explored above. It is important to note that these factors and their interactions can change during this period of growth, development and maturation. Taking a detailed sleep history enables these factors to be established and then further assessed as indicated; key points to discuss are shown in Box 1. Undertaking at least part of the consultation with only the young person (without parents or guardians present) and with the assurance of confidentiality is good clinical practice for this age group.

A full sleep history may not always be possible and therefore shorter tools such as the BEARS ( $\mathrm{B}=$ bedtime issues, $\mathrm{E}=$ excessive daytime sleepiness, $\mathrm{A}=$ night awakenings, $\mathrm{R}=$ regularity and duration of sleep, $\mathrm{S}=$ snoring) screening tool (Table 1) or even the use of the HEEADSSS (home environment, education and employment, eating, peer-related activities, drugs, sexuality, suicide/depression, safety) psychosocial tool can be a useful first assessment in this age group. ${ }^{42,43}$ A more detailed history can then be taken if a specific problem is identified.

Sleep education is often the first step required for successful management and as such a number of studies have looked at

\section{Box 1. Key points of a sleep history}
$>$ Habitual bedtime and rise time
$>$ Sleep duration
$>$ Sleepiness
$>$ Difficulties falling asleep (sleep-onset latency)
$>$ Number of night wakings
Number of daytime naps
Subjective opinion
Fatigue
Other sleep problems
$>$ Beliefs
Drug use
Caffeine/stimulant use
School day versus weekend (sleep irregularities)
School achievement
Competing demands
Bedroom environment
Smart phone/computer use
Injuries
Driving 
Table 1. BEARS screening tool ${ }^{42}$

$\begin{array}{ll}\begin{array}{l}\text { Categories } \\ \text { Bedtime problems }\end{array} & \begin{array}{l}\text { Example questions } \\ \text { Do you have any problems falling asleep } \\ \text { at bedtime? }\end{array} \\ \begin{array}{ll}\text { Excessive daytime } & \text { Do you feel sleepy a lot during the day? } \\ \text { sleepiness } & \text { At school? While driving? } \\ \text { Awakenings during } & \text { Do you wake up a lot during the night? } \\ \text { the night } & \\ \begin{array}{l}\text { Regularity and } \\ \text { duration of sleep }\end{array} & \begin{array}{l}\text { What time do you go to bed on a school } \\ \text { night? } \text { you usually get? }\end{array} \\ \begin{array}{ll}\text { Sleep disordered } \\ \text { breathing }\end{array} & \text { Ask family members about snoring. }\end{array} \\ \end{array}$

the role of sleep education in adolescents, often in a school environment. These studies include some that were aiming to disseminate knowledge alongside those attempting to effect a change in behaviour. A review of these by Blunden and Ridgney, demonstrated the importance of including others, such as parents, as sleep is affected by both cultural and environmental factors within the home. ${ }^{44}$ Importantly, it was noted that a generic sleep programme would be unlikely to benefit all individuals. Identifying specific problems was a more useful approach and imparting knowledge alone was not enough to effect a change in behaviour.

Exploring the young person's (and where appropriate, the family's) understanding of sleep and discussing sleep hygiene is a useful first management strategy. The concept of sleep hygiene relates to establishing a set regimen that in turn encourages a regular sleep and wake pattern. Important aspects of this include avoiding daytime naps, avoiding stimulant usage (including caffeine) in the afternoon, only using the bed for sleeping, ensuring a calm and relaxing sleep environment, encouraging exposure to natural daylight and encouraging exercise. Sleep hygiene advice is easily accessed via a number of online resources, some of which are shown in Table 2.

The use of handheld media devices is a now a significant part of life, particularly for AYAs, and is therefore worth separate

Table 2. Resources

$\begin{array}{ll}\begin{array}{l}\text { The Sleep Council } \\ \text { NHS Choices }\end{array} & \begin{array}{l}\text { www.sleepcouncil.org.uk/how-to- } \\ \text { sleep/sleeping-tips-for-teenagers/ } \\ \text { www.nhs.uk/Livewell/Childrenssleep/ } \\ \text { Pages/teensleeptips.aspx }\end{array} \\ \begin{array}{l}\text { Royal College of } \\ \text { Psychiatrists }\end{array} & \begin{array}{l}\text { ww.rcpsych.ac.uk/healthadvice/ } \\ \text { parentsandyouthinfo/parentscarers/ } \\ \text { sleepproblems.aspx }\end{array} \\ \begin{array}{l}\text { Sleep Scotland } \\ \text { The Children's Sleep } \\ \text { Charity }\end{array} & \text { www.thechildrenssleepcharity.org.uk/ } \\ \begin{array}{l}\text { The REST (resources } \\ \text { for effective sleep } \\ \text { treatment) Project }\end{array} & \text { http://elearning.restproject.org.uk/ }\end{array}$

consideration. There has been increasing interest in the impact that the use of devices such as smart phones and tablets have on sleep within this population group. A 2016 systematic review has confirmed that bedtime use and access to these devices were significantly associated with inadequate sleep quality, poor sleep quality and excessive daytime sleepiness. ${ }^{45}$ Exploring usage of such devices is therefore important if a sleep problem has been identified. Good sleep hygiene advice would include not having these devices within the bedroom, thus reducing the temptation to use them.

\section{Conclusions}

Sleep has an important role to play in the health of adolescents and young adults, both in the short and longer term. A number of adverse outcomes have been shown to be associated with poor sleep within this population. Improved recognition of its importance and the assessment of sleep in routine clinical practice can help to identify and manage problems before longer-term consequences develop. The use of simple screening tools can help and there are a number of online resources that can guide both the clinician and patient.

\section{Conflicts of interest}

The authors have no conflicts of interest to declare.

\section{References}

1 Kopasz M, Loessl B, Hornyak M et al. Sleep and memory in healthy children and adolescents - a critical review. Sleep Med Rev 2010;14:167-77.

2 Opp MR, Krueger JM. Sleep and immunity: a growing field with clinical impact. Brain Behav Immun 2015;47:1-3.

3 Cappuccio FP, D'Elia L, Strazzullo P, Miller MA. Sleep duration and all-cause mortality: a systematic review and meta-analysis of prospective studies. Sleep 2010;33:585-92.

4 Chandola T, Ferrie JE, Perski A, Akbaraly T, Marmot MG. The effect of short sleep duration on coronary heart disease risk is greatest among those with sleep disturbance: a prospective study from the Whitehall II cohort. Sleep 2010;33:739-44.

5 Cain N, Gradisar M. Electronic media use and sleep in school-aged children and adolescents: a review. Sleep Med 2010;11:735-42.

6 Lemola S, Perkinson-Gloor N, Brand S, Dewald-Kaufmann JF, Grob A. Adolescents' electronic media use at night, sleep disturbance, and depressive symptoms in the smartphone age. $J$ Youth Adolesc 2015;44:405-18.

7 Brooks F, Magnusson J, Klemera E, Spencer N, Smeeton N. HBSC England National Report: health behaviour in school-aged children (HBSC): World Health Organization Collaborative Cross National Study. Hatfield, UK: University of Hertfordshire, 2015.

8 Dregan A, Armstrong D. Adolescence sleep disturbances as predictors of adulthood sleep disturbances-a cohort study. J Adolesc Health 2010;46:482-7.

9 Steinberg L. A behavioral scientist looks at the science of adolescent brain development. Brain Cogn 2010;72:160-4.

10 Colrain IM, Baker FC. Changes in sleep as a function of adolescent development. Neuropsychol Rev 2011;21:5-21.

11 Crowley SJ, Acebo C, Carskadon MA. Sleep, circadian rhythms, and delayed phase in adolescence. Sleep Med 2007;8:602-12.

12 Figueiro M. Delayed sleep phase disorder: clinical perspective with a focus on light therapy. Nat Sci Sleep 2016;91-106.

13 Kotagal S, Pianosi P. Sleep disorders in children and adolescents. BMJ 2006;332:828-32. 
14 Cappuccio FP, Taggart FM, Kandala N-B et al. Meta-analysis of short sleep duration and obesity in children and adults. Sleep 2008;31:619-26.

15 Nielsen LS, Danielsen KV, Sørensen TIA. Short sleep duration as a possible cause of obesity: critical analysis of the epidemiological evidence: short sleep as possible cause of obesity. Obes Rev 2011;12:78-92.

16 Garaulet M, Ortega FB, Ruiz JR et al. Short sleep duration is associated with increased obesity markers in European adolescents: effect of physical activity and dietary habits. The HELENA study. Int $J$ Obes 2011;35:1308-17.

17 Noland H, Price JH, Dake J, Telljohann SK. Adolescents' sleep behaviors and perceptions of sleep. J Sch Health 2009;79:224-30.

18 Leproult R, Van Cauter E. Role of sleep and sleep loss in hormonal release and metabolism. In: Loche S, Cappa M, Ghizzoni L, Maghnie M, Savage MO, eds. Paediatric neuroendocrinology. Basel: KARGER, 2009:11-21.

19 Matthews KA, Dahl RE, Owens JF, Lee L, Hall M. Sleep duration and insulin resistance in healthy black and white adolescents. Sleep 2012;35:1353-8.

20 Chorney DB, Detweiler MF, Morris TL, Kuhn BR. The Interplay of sleep disturbance, anxiety, and depression in children. J Pediatr Psychol 2007;33:339-48.

21 Gregory AM, Sadeh A. Sleep, emotional and behavioral difficulties in children and adolescents. Sleep Med Rev 2012;16:129-36.

22 Gangwisch JE, Babiss LA, Malaspina D et al. Earlier parental set bedtimes as a protective factor against depression and suicidal ideation. Sleep 2010;33:97-106.

23 McKnight-Eily LR, Eaton DK, Lowry R et al. Relationships between hours of sleep and health-risk behaviors in US adolescent students. Prev Med 2011;53:271-3.

24 O'Brien EM, Mindell JA. Sleep and risk-taking behavior in adolescents. Behav Sleep Med 2005;3:113-33.

25 Luntamo T, Sourander A, Rihko M et al. Psychosocial determinants of headache, abdominal pain, and sleep problems in a community sample of Finnish adolescents. Eur Child Adolesc Psychiatry 2012;21:301-13.

26 Schrimpf M, Liegl G, Boeckle M et al. The effect of sleep deprivation on pain perception in healthy subjects: a meta-analysis. Sleep Med 2015;16:1313-20.

27 Bellini B, Panunzi S, Bruni O, Guidetti V. Headache and sleep in children. Curr Pain Headache Rep 2013;17:335.

28 Pecor K, Kang L, Henderson M et al. Sleep health, messaging, headaches, and academic performance in high school students. Brain Dev 2016;38:548-53.

29 Bellini B, Arruda M, Cescut A et al. Headache and comorbidity in children and adolescents. J Headache Pain 2013;14:79.

30 Dosi C, Figura M, Ferri R, Bruni O. Sleep and headache. Semin Pediatr Neurol 2015;22:105-12.

31 Butbul Aviel Y, Stremler R, Benseler SM et al. Sleep and fatigue and the relationship to pain, disease activity and quality of life in juvenile idiopathic arthritis and juvenile dermatomyositis. Rheumatology 2011;50:2051-60.
32 Stinson JN, Hayden JA, Ahola Kohut S et al. Sleep problems and associated factors in children with juvenile idiopathic arthritis: a systematic review. Pediatr Rheumatol Online J 2014;12:19.

33 Palermo TM, Wilson AC, Lewandowski AS, Toliver-Sokol M, Murray CB. Behavioral and psychosocial factors associated with insomnia in adolescents with chronic pain. Pain 2011;152:89-94.

34 Albon L, Vaughan L. Adolescents and young adults on the acute medical unit: how might we do it better? Clin Med 2014;14:618-22.

35 Lewandowski AS, Ward TM, Palermo TM. Sleep problems in children and adolescents with common medical conditions. Pediatr Clin North Am 2011;58:699-713.

36 Findlay SM. The tired teen: a review of the assessment and management of the adolescent with sleepiness and fatigue. Paediatr Child Health 2008;13:37-42.

37 Rimes KA, Goodman R, Hotopf $\mathrm{M}$ et al. Incidence, prognosis, and risk factors for fatigue and chronic fatigue syndrome in adolescents: a prospective community study. Pediatrics 2007;119:e603-9.

38 National Institute for Health and Care Excellence. Chronic fatigue syndrome/myalgic encephalomyelitis (or encephalopathy): diagnosis and management. NICE clinical guideline No 52. London: NICE, 2007.

39 Wolfe I, Macfarlane A, Donkin A, Marmot M, Viner R. Why children die: death in infants, children and young people in the UK. Part A. London: Royal College of Paediatrics and Child Health, 2014.

40 Flatley D, Reyner LA, Horne JA. Sleep-related crashes on sections of different road types in the UK (1995-2001). London: Department for Transport, 2004.

41 Patton GC, Coffey C, Cappa C et al. Health of the world's adolescents: a synthesis of internationally comparable data. Lancet 2012;379:1665-75.

42 Owens JA, Dalzell V. Use of the 'BEARS' sleep screening tool in a pediatric residents' continuity clinic: a pilot study. Sleep Med 2005;6:63-9.

43 Goldenring J, Rosen D. Getting into adolescent heads: an essential update. Contemp Pediatr 2004;21:64-90.

44 Blunden S, Rigney G. Lessons learned from sleep education in schools: a review of dos and don'ts. J Clin Sleep Med 2015;11:67180.

45 Carter B, Rees P, Hale L, Bhattacharjee D, Paradkar MS. Association Between portable screen-based media device access or use and sleep outcomes: a systematic review and meta-analysis. JAMA Pediatr 2016;170:1202-8.

Address for correspondence: Dr Ellen Bruce, The Kellgren Centre for Rheumatology, Central Manchester University Hospitals NHS Foundation Trust, Oxford Road, Manchester M13 9WL, UK.

Email: ellen.bruce@cmft.nhs.uk 